\title{
Three-dimensional investigation of liquid film structure at the initial area of annular-dispersed flow
}

\author{
Sergey Alekseenko ${ }^{1,2}$, Andrey Cherdantsev ${ }^{1,2}$, Mikhail Cherdantsev ${ }^{1}$, Sergey Isaenkov ${ }^{1}$ and Dmitriy \\ Markovich ${ }^{1,2}$ \\ ${ }^{1}$ Kutateladze Institute of Thermophysics SB RAS, Lavrentiev ave., 1 , Novosibirsk, 630090, Russia \\ ${ }^{2}$ Novosibirsk State University, Pirogov str., 2, Novosibirsk, 630090, Russia
}

\begin{abstract}
Initial stage of downward flow of gas-sheared liquid film in a vertical rectangular duct was studied using brightness-based laser-induced fluorescence technique. Measurements were resolved along both longitudinal and transverse coordinates and time. The initial highfrequency waves which are formed at the inlet were found to be two-dimensional. These waves are promptly broken into localised horseshoe-shaped waves which merge downstream to form large-scale quasi-2D disturbance waves. Peculiarities of three-dimensional evolution of waves of different types were studied in a wide range of flow parameters.
\end{abstract}

\section{Introduction}

In annular-dispersed flow liquid film flows on the inner wall of the pipe sheared by high-velocity gas stream flowing in the center of the pipe. In such a flow large-scale disturbance waves are formed on film surface. Presence of disturbance waves is necessary for liquid entrainment into the gas core. These waves cause enhancement of pressure drop and heat transfer. Modeling of wavy structure of liquid film is complicated by multi-scale nonlinear wavy processes and strong turbulence in both phases. To verify the existing models and to create new models detailed experimental information describing the interactions of waves is required.

Experiments by local conductivity probes showed that near the inlet film surface is covered by high-frequency small-amplitude waves [1]. In recent paper [2] spatiotemporal evolution of waves in one longitudinal section of $15 \mathrm{~mm}$ pipe was studied near the inlet using laser-induced fluorescence technique. It was concluded that disturbance waves are formed due to multiple coalescence of initial high-frequency waves that appear due to Kelvin-Helmholtz instability.

The goal of the present paper is to investigate the process of formation of disturbance waves in three-dimensional approach, i.e., with measurements of local film thickness resolved in both longitudinal and transverse coordinates and time.

\section{Experimental setup and measurement technique}

In pipe geometry obtaining 3D film thickness profile over the whole wetted perimeter requires an experimental system consisting of several cameras, which is expensive and complicated. Besides, it would be difficult to completely eliminate optical distortions appearing due to the curvature of the pipe [3]. It was recently shown [4] that the wavy structure of gas-sheared films is qualitatively the 
same in channels of different shape, size and orientation. Thus, it was considered that investigation of 3D-evolution of waves near the inlet would be more comprehensive in a rectangular duct which provides clear optical access to the whole width of the channel (Fig. 1). The duct's dimensions are $700 \mathrm{~mm}$ (length) by $50 \mathrm{~mm}$ (width) by $5 \mathrm{~mm}$ (height). Liquid film is formed by $0.5 \mathrm{~mm}$ width tangential slot between one of the long walls and a thin stainless steel plate.

Figure 1. A sketch of measurement section.

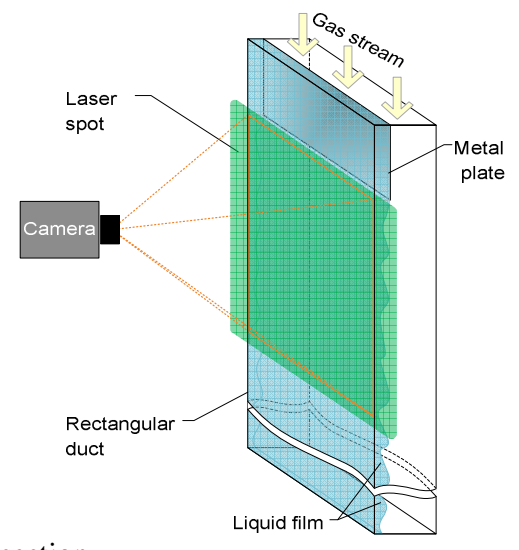

Brightness-based laser-induced fluorescence (LIF) method was used for film thickness measurements. Fluorescent dye (Rhodamine-6G) was dissolved in working liquid. The area of measurements was illuminated by continuous $2 \mathrm{~W}$ laser with wavelength of $532 \mathrm{~nm}$. Reemitted fluorescent light was recorded by high-speed camera and recalculated into local film thickness. Detailed description of this method can be found in [3]. In present experiments, frame rate of high speed camera is $1 \mathrm{KHz}$; spatial resolution is $0.2 \mathrm{~mm} /$ pixel. The size of the investigated area is $50 \mathrm{~mm}$ in transverse direction, $y$, and $200 \mathrm{~mm}$ in longitudinal direction, $x$.

\section{Results and discussion}

According to $x$-t-representation (Fig. 2a), the disturbance waves are formed due to coalescence of initial waves which appear just after the inlet. This process turns out to be more sophisticated in 3Dapproach. Fig. 2b shows instantaneous shape of film surface for time moment marked by the dashed line in Fig. 2a. The initial waves are two-dimensional near the inlet, but farther downstream they are broken into small-scale transversely localised horseshoe-shaped waves. Further downstream avalanche-like merging of large number of these 3D waves occurs to form the disturbance waves. The latter are characterised by much larger transverse size which grows downstream approaching to that of the duct.

Thus, the degree of two-dimensionality - or transverse coherence - of film surface can be described as follows. It is very high near the inlet due to two-dimensional character of KelvinHelmholtz instability; when the initial waves develop, increasing in amplitude and nonlinearity, transverse instability of the waves leads to complete loss of two-dimensionality. But, due to action of gas shear, coalescence of small waves produces wider waves which are surprisingly stable to transverse perturbations and continue to absorb the smaller waves and grow in transverse size. Supposedly, this stability is explained by much longer wavelength in longitudinal direction in comparison to that of the initial waves. As a result, the transverse coherence is regained gradually. This is in agreement to the observations of the paper [1], though further downstream this increase may also occur due to coalescence of narrower disturbance waves to form wider waves, as it was noticed in [4]. 


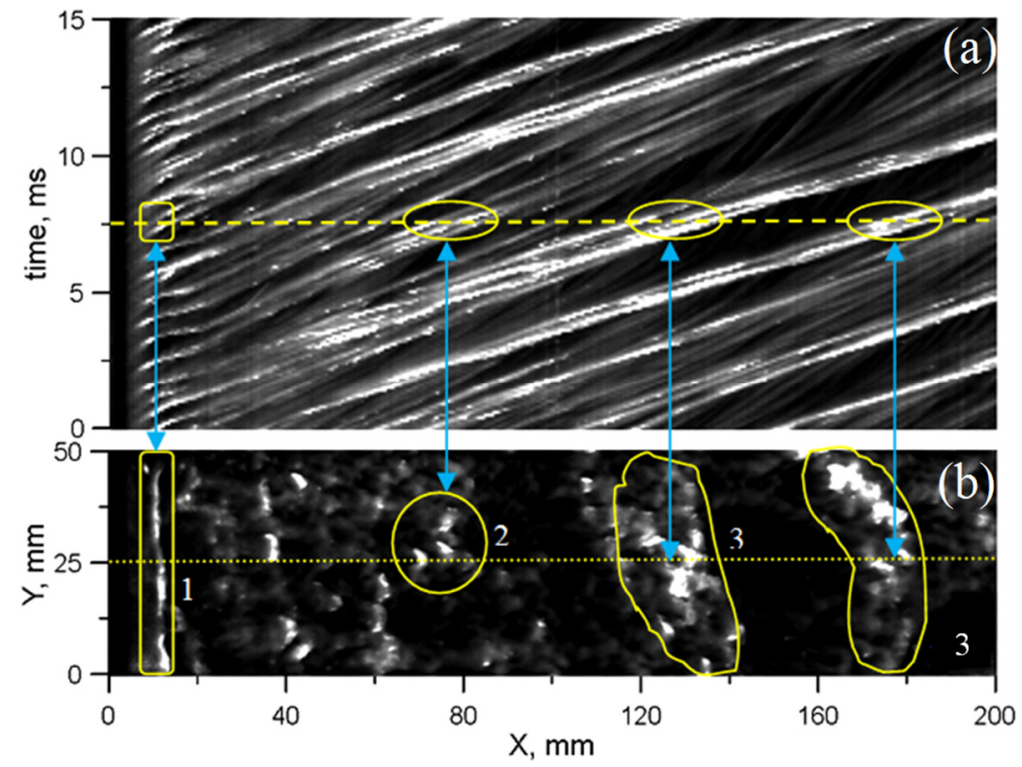

Figure 2. Formation of disturbance waves in $x-t$ (a) and $x-y$ (b) representation. Local brightness of the image is directly proportional to local film thickness. $R_{L}=300, V_{g}=26 \mathrm{~m} / \mathrm{s}$. $1-$ initial 2D waves, $2-3 \mathrm{D}$ waves, $3-$ disturbance waves.

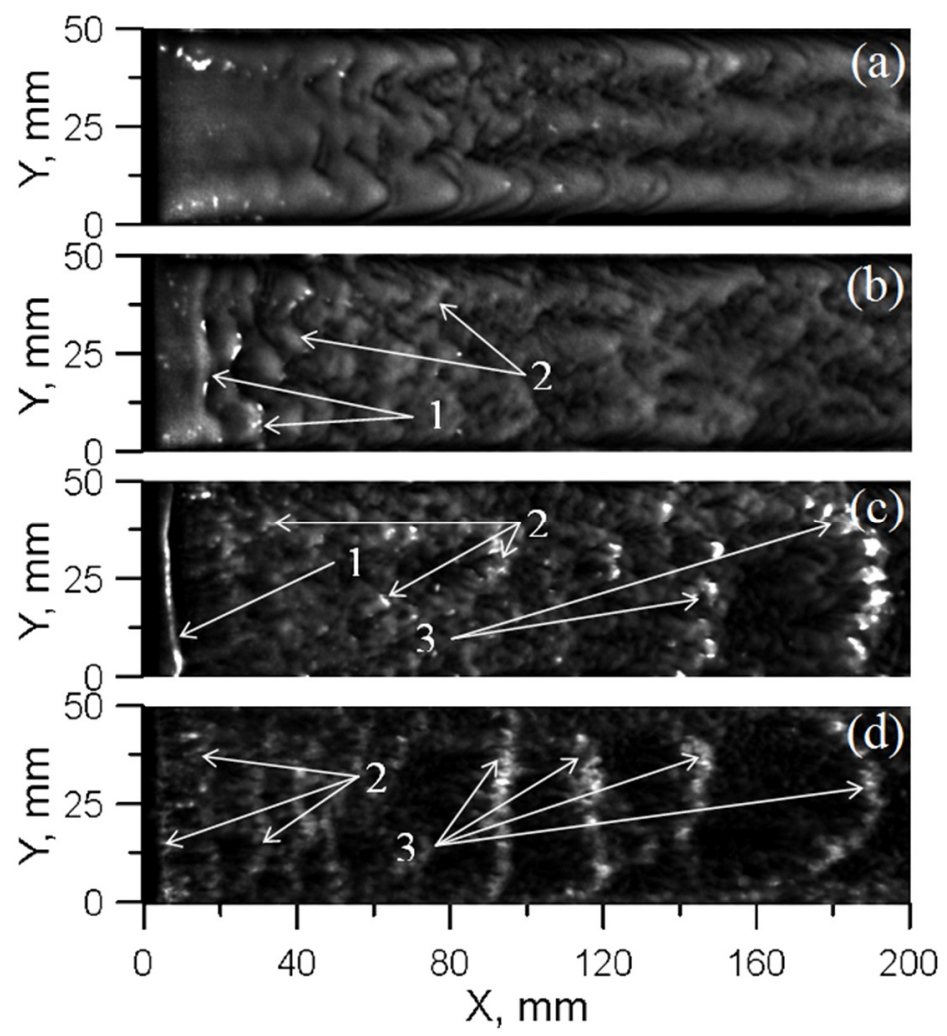

Figure 3. Instantaneous three-dimensional film thickness profiles for $R_{L}=220 . V_{g}=14 \mathrm{~m} / \mathrm{s}$ (a), $V_{g}=18 \mathrm{~m} / \mathrm{s}$ (b), $V_{g}=26 \mathrm{~m} / \mathrm{s}(\mathrm{c}), \mathrm{Vg}=43 \mathrm{~m} / \mathrm{s}(\mathrm{d}) .1$ - initial 2D waves, $2-3 \mathrm{D}$ waves, 3 - disturbance waves. 
Fig. 3 shows examples of instantaneous shapes of film surface at different gas velocities, $V_{g}$. At low $V_{g}$ (Fig. 3a) the film surface is covered by longitudinally-oriented rivulets. It was recently observed [5] that this structure is also typical for adiabatic falling films. Increasing gas speed makes the film thickness more uniform which is inherent to annular flow. Length over which the initial waves are two-dimensional shortens as $V_{g}$ increases (Fig. 3b-d). The increase leads to stronger rates of amplitude growth of the initial waves; hence, the transverse instability becomes significant earlier. At the highest gas speeds (Fig. 3d), the 2D waves can hardly be noticed; they are broken into 3D waves immediately after the inlet. Transition from the 3D waves to disturbance waves also occurs closer to the inlet at larger $V_{g}$. At low $V_{g}$ (Fig 3a-b) the disturbance waves are not observed within the area of interrogation, whilst at the largest $V_{g}$ the disturbance waves appear at the distance of less than $100 \mathrm{~mm}$ from the inlet. This effect can be explained by larger number of 3D waves with larger scatter in velocity and time separation, which increases probability of coalescence.

\section{Conclusions}

The mechanism of formation of disturbance waves, discovered recently in frames of 2D-approach, was confirmed during the present $3 \mathrm{D}$ experiments in a duct of a different shape. It was found that during the initial stages of flow of gas-sheared liquid films the transverse coherence of waves shows non-monotonic behaviour due to cascade of events dominating at certain stages, namely, twodimensional Kelvin-Helmholtz instability, three-dimensional transverse instability and formation of large-scale quasi-2D disturbance waves. Range of distances from the inlet over which each stage is achieved depends on flow parameters, primarily, on the gas velocity. The gas flow also straightens the film in transverse direction, suppressing the formation of longitudinally oriented rivulets, but enhances formation of transversely oriented disturbance waves.

\section{Acknowledgements}

The work was supported by Russian Scientific Foundation (project 16-19-10449).

\section{References}

1. Y. Zhao, C.N. Markides, O.K. Matar, G.F. Hewitt, Int. J. Multiphase Flow, 55, 111-129 (2013).

2. S.V. Alekseenko, A.V. Cherdantsev, M.V. Cherdantsev, S.V. Isaenkov, D.M. Markovich, Int. J. Multiphase Flow, 77, 65-75 (2015).

3. S. Alekseenko, A. Cherdantsev, M. Cherdantsev, S. Isaenkov, S. Kharlamov, D. Markovich, Exp. Fluids, 53, 77-89 (2012).

4. A.V. Cherdantsev, D.B. Hann, B.J. Azzopardi, Int. J. Multiphase Flow, 67, 52-64 (2014).

5. S.M. Kharlamov, V.V. Guzanov, A.V. Bobylev, S.V. Alekseenko, D.M. Markovich, Phys. Fluids, 27, 114106 (2015). 